\title{
Akademisierte Pflegekräfte
}

\author{
Potenziale sollten besser genutzt werden
}

Mit der Akademisierung der Pflege sind in Deutschland Qualifikationsprofile entstanden, die die gesundheitliche Versorgung der Bevölkerung verbessern können. Aktuell gibt es etwa 10.000 Studierende in den Pflegewissenschaftlichen Studiengängen und ca. 1.500 Absolventen jährlich. Ein Potenzial, das noch immer viel zu wenig genutzt wird. In Berlin erläuterten Pflegewissenschaftler kürzlich, weshalb akademisierte Pflegekräfte besser in die Patientenversorgung integriert werden sollten.

Bedarfe generieren sich aus dem demographischen Wandel, der zunehmenden Urbanisierung sowie Veränderungen des Krankheitsspektrums. Diese Faktoren machen, einer Ausgangsanalyse von Prof. Renate Stemmer, Katholische Hochschule Mainz und Vorsitzende der Deutschen Gesellschaft für Pflegewissenschaft, zufolge, eine Integration akademisch ausgebildeter Pflegemitarbeiter in die Gesundheitsversorgung zum Gebot der Stunde. Multimorbide Patienten und Menschen mit chronischen Erkrankungen bedürfen einer differenzierten Pflege und Betreuung. Untersuchungen belegen eine verbesserte Patientenversorgung durch hochschulisch ausgebildete Pflegende, die wissenschaftliches Denken in das pflegerische Handeln einbeziehen. „Um spürbare Auswirkungen dieser Kompetenzen auf die Versorgungsqualität $\mathrm{zu}$ erreichen, ist allerdings ein quantitativer Schwellenwert in der Praxis erforderlich“, so Stemmer. Die Absolventen würden zwar viele berufliche Optionen finden, aber viel zu selten in der Patientenversorgung eingesetzt.

Nach Ansicht von Prof. Petra Weber, Vorstand der Dekanekonferenz Pflege, ist es daher unerlässlich, alle Akteure im Gesundheitswesen zusammenzubringen. Sie fordert einen Diskurs zwischen Hochschulen, Politik und Unternehmen. Nur so seien Verbesserungen in der Versorgung möglich. Klientennahe Tätigkeiten sind nach Auffassung von Weber in den Unternehmen konzeptionell noch zu wenig entwickelt. „Insbesondere Menschen mit chronischen Krankheitsverläufen profitieren von akademisch qualifizierten Pflegenden, weil ihre Handlungsperspektive die medizinische Seite und die individuelle Lebenssituation der betroffenen Menschen verbindet", so Weber.

Die deutsche Pflegeausbildung sei schon immer ein Sonderweg innerhalb Europas gewesen, betonte Franz Wagner, Bundesgeschäftsführer des Deutschen Berufsverbands für Pflegeberufe (DBfK). Auch das Bild der Berufskompetenzen sei hier nach wie vor geprägt von Arztassistenz. „Die Einsicht, dass man akademisch ausgebildete Pflegekräfte für die Leistungsfähigkeit des Systems braucht, ist in der Politik noch nicht angekommen", stellte er ernüchtert fest. Die Akademisierung würde noch zu sehr als Professionalisierungsprojekt wahrgenommen, ihre Vorteile nicht erkannt und daher auch nicht genutzt.
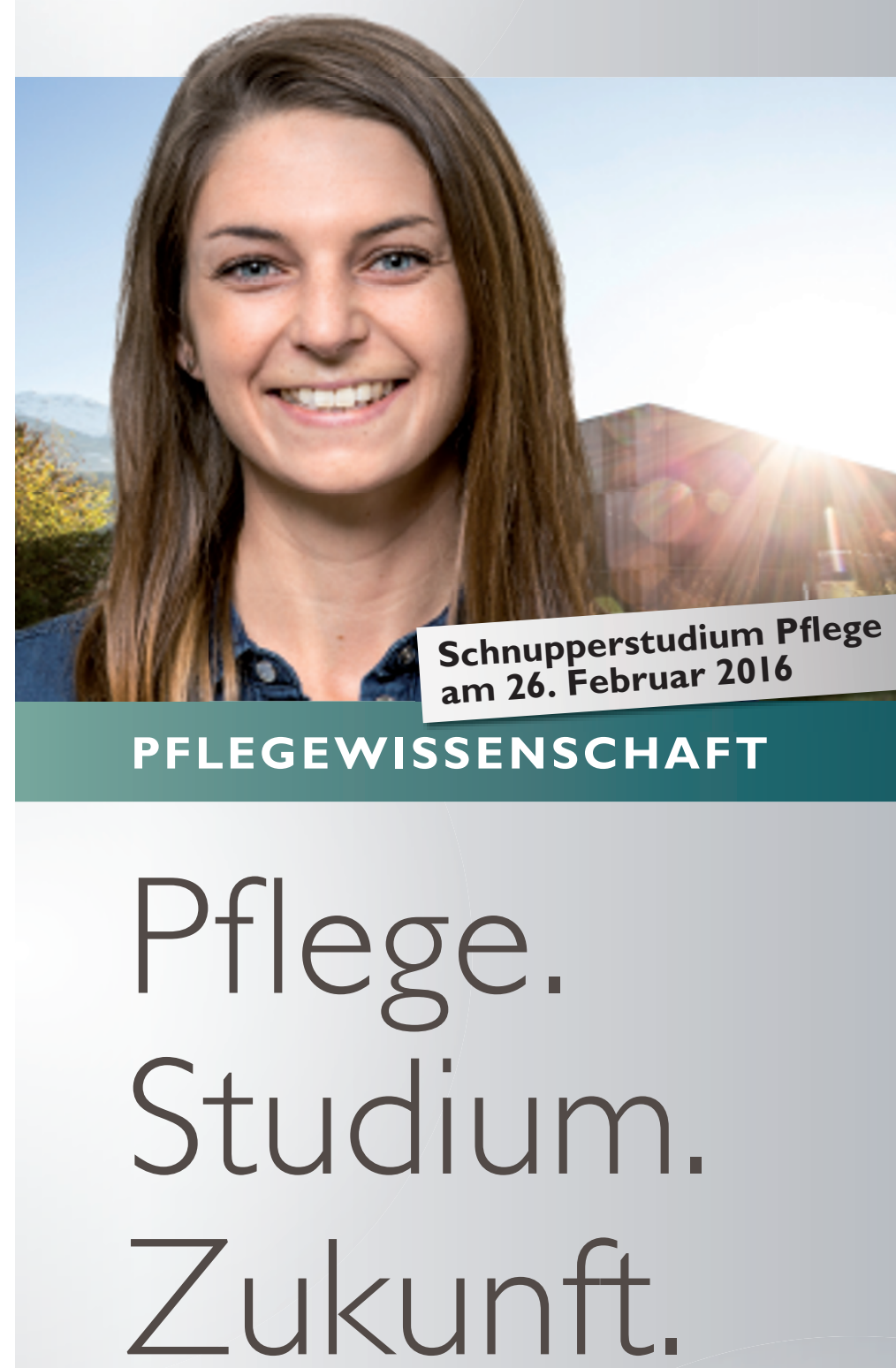

Pflegewissenschaft für diplomierte Pflegepersonen als Bachelor-, Master- oder Doktorat-Studium am modernen Uni-Campus in Hall in Tirol.

\section{Ab sofort: \\ — Universitätslehrgang „Führungsaufgaben in der Gesundheits und Krankenpflege“ \\ _ Universitätslehrgang „Lehraufgaben in der Gesundheits- und Krankenpflege“}

Kontakt: lehre@umit.at I www.umit.at

Quelle: Pressekonferenz Fachtagung „Die Zukunft der Gesundheitsversorgung der Beitrag akademisierter Pflegekräfte“, Veranstalter: Deutsche Gesellschaft für Pflegewissenschaft e.V. und Dekanekonferenz Pflegewissenschaft e.V, Berlin, 5. November 2015 . 